\title{
Probing the Redox Selectivity on Au@Pd and Au@Pt Bimetallic Nanoplates by Tip-Enhanced Raman Spectroscopy (TERS)
}

\author{
Zhandong Li', and Dmitry Kurouski ${ }^{* 1,2}$ \\ ${ }^{1}$ Department of Biochemistry and Biophysics, Texas A\&M University, College Station, Texas 77843, United States \\ ${ }^{2}$ The Institute for Quantum Science and Engineering, Texas A\&M University, College Station, Texas, 77843, United \\ States
}

Email: dkurouski@tamu.edu

\section{Contents}

Experimental Details.

SI1. AFM images and height profile of Au@PtNPs.

SI2. SEM images of AuNPs, Au@PtNPs and Au@PdNPs.

SI3. AFM images and height profile of 15-nm AuNPs.

SI4. AFM images and height profiles of AuNPs prepared by isotropical growth.

SI5. AFM images and height profile of Au@PdNPs.

SI6. AFM images and statistical height analysis of AuNPs, Au@PtNPs and Au@PdNPs.

SI7. UV-vis-NIR of absorption spectra of AuNPs, Au@PtNPs and Au@PdNPs.

SI8. More TERS spectra of catalyzing MPM on Au@PtNPs.

SI9. SERS spectra of MPM, MBA and TP.

SI10. Low resolution TERS mapping of MPM/MBA on AuNPs, Au@PtNPs and Au@PdNPs.

SI11. More TERS spectra of catalyzing MBA on Au@PdNPs.

SI12. High resolution AFM images and height distribution of Au@PtNPs and Au@PdNPs.

SI13. High resolution AFM image and TERS images of Au@PdNPs.

SI14. Control experiments showing TERS of MPM on Au@PdNPs and MBA on Au@PtNPs.

SI15. Kinetic catalyzation process of MPM on Au@PtNPs and MBA on Au@PdNPs.

SI16. Rate constant of MPM-MBA photo-redox at different spots.

SI17 More TERS spectra of catalyzing MPM/MBA on AuNPs.

Table S1. Kinetic parameters of MPM oxidation and MBA reduction. 


\section{Experimental Details.}

\section{Chemicals}

Gold (III) chloride trihydrate $\left(\mathrm{HAuCl}_{4} \cdot 3 \mathrm{H}_{2} \mathrm{O}, 99.9 \%\right)$, Palladium (II) chloride solution $\left(\mathrm{H}_{2} \mathrm{PdCl}_{4}\right)$, Chloroplatinic acid solution $\left(\mathrm{H}_{2} \mathrm{PtCl}_{6}\right)$, hexadecyltrimethylammonium bromide (CTAB, 99\%), 4-Mercapto-phenyl)-methanol (MPM), 4Mercaptobenzoic Acid (MBA), sodium hydroxide (NaOH, 98\%), potassium iodide (KI, 99\%), L-ascorbic acid (AA, 99\%), sodium borohydride $\left(\mathrm{NaBH}_{4}, 99 \%\right)$ were purchased from Sigma-Aldrich (St. Louis, MO). Sodium citrate dihydrate (NaCit, 99\%) was purchased from Fisher scientific (Waltham, MA). Ethanol was purchased from Decon Labs (King of Prussia, PA). All chemicals were used as received without purification.

\section{Preparation of the Au, Au@Pd and Au@Pt nanoplates (AuNPs, Au@PdNPs and Au@PtNPs)}

The AuNPs were prepared by first seed-mediated growth method following by the isotropical growth. Au seeds solution was first prepared by add $0.01 \mathrm{M}, 1 \mathrm{~mL}$ of $\mathrm{HAuCl}_{4}$ and $0.01 \mathrm{M}, 1 \mathrm{~mL}$ of Na-Cit solutions into $36 \mathrm{ml}$ of water. Then, under vigorous stirring, $0.1 \mathrm{M}, 1 \mathrm{~mL}$ of ice-cold $\mathrm{NaBH}_{4}$ solution was introduced and keep stirring for 2 min. Next, the mixture solution was kept at room temperature without disturbing, aging for 2-6 h. Au nanoplates (AuNPs) seed solution with $\sim 15$ nm thickness with triangle or hexagonal (See Figure. S1) was then prepared by three-step seed-mediated growth of the Au seeds after aging. Briefly, the growing solutions 1, 2 and 3 were prepared as following methods. Firstly, growing solution 1 and 2 were prepared the same where $0.01 \mathrm{M}, 0.25 \mathrm{~mL}$ of $\mathrm{HAuCl}_{4}, 0.1 \mathrm{M}, 0.05 \mathrm{~mL}$ of $\mathrm{NaOH}, 0.01 \mathrm{M}, 0.05 \mathrm{~mL}$ of $\mathrm{KI}$, and 0.1 M, $0.05 \mathrm{~mL}$ of Ascorbic Acid (AA) was added into $0.05 \mathrm{M}, 9 \mathrm{~mL}$ of CTAB solution by order. Then, the third growing solution was prepared similarly by introducing $0.01 \mathrm{M}, 2.5 \mathrm{~mL}$ of $\mathrm{HAuCl}_{4}, 0.1 \mathrm{M}, 0.5 \mathrm{~mL}$ of NaOH, $0.01 \mathrm{M}, 0.5 \mathrm{~mL}$ of $\mathrm{KI}$, and $0.1 \mathrm{M}, 0.5 \mathrm{~mL}$ of AA into $0.05 \mathrm{M}, 90 \mathrm{~mL}$ of CTAB solution. Subsequently, $1 \mathrm{~mL}$ of the Au seeds stocking solution was added into growing solution 1, followed by gently shaking for $5 \mathrm{~s}$. Then, $1 \mathrm{~mL}$ of the Au seeds and growing solution 1 mixture were added to the growth solution 2, followed by gently shaking for another $5 \mathrm{~s}$. Finally, all of the Au seeds and growth solution 1, 2 mixture were added to the growing solution 3 following by gently shaking for $5 \mathrm{~s}$. The final solution was then kept at room temperature without any disturbing for overnight. Then, the $\sim 15 \mathrm{~nm}$ thickness AuNPs seeds were collected by precipitating at $5000 \mathrm{rpm}$ for $2 \mathrm{~min}$ and dissolved in $5 \mathrm{~mL}$ of CTAB solution for the next step of growth. The thicker Au nanoplates (AuNPs) was then synthesized by conducting isotropical growth on the $\sim 15$ nm thickness AuNPs in diluted CTAB solution. For isotropical growth, briefly, the growth solution was prepared by mixing $0.25 \mathrm{M}, 1$ $\mathrm{mL}_{\text {of }} \mathrm{HAuCl}_{4}, 0.1 \mathrm{M}, 0.055 \mathrm{~mL}$ of AA and water $(8 \mathrm{~mL})$ with $0.1 \mathrm{M}, 1 \mathrm{~mL}$ of CTAB solution by order. Then, the isotropical growth reaction was initialized by adding $0.3 \mathrm{ml}$ of the AuNPs seeds solution (15 $\mathrm{nm})$ into the above freshly prepared isotropical growth solution. After this step, the expecting thickness of AuNPs was at least $60 \mathrm{~nm}$. Next, Au@PtNPs and Au@PdNPs were both prepared by this recipe, $250 \mu 1$ of the AuNPs $(60 \mathrm{~nm})$ stock solution was first 
mixed with $60 \mu \mathrm{L}$ of $20 \mathrm{mM}$ AA. Subsequently, the solution was brought to be mixed by vortexing for $10 \mathrm{~s}$. Next, $15 \mu \mathrm{L}$, $10 \mathrm{mM}$ of $\mathrm{H}_{2} \mathrm{PtCl}_{6}$ solution was introduced following by vortexing for $10 \mathrm{~s}$ again. The solution was kept at room temperature for 1 hours without disturbing until the completion of bimetallic nano-plates growth. For purification, the solution was centrifuged at 8000 rcf 2 min for twice. Finally, after removal of the supernatant, the Au@PtNPs were dissolved in $1.0 \mathrm{~mL}$ of water and sonicated for $20 \mathrm{~s}$. Resembly, $30 \mu \mathrm{L}, 10 \mathrm{mM}$ of $\mathrm{H}_{2} \mathrm{PtCl}_{4}$ was introduced to the mixture of AuNPs $(60 \mathrm{~nm})$ stock solution and $60 \mu \mathrm{L}$ of $20 \mathrm{mM}$ AA following the same purification process and re-dispersed in 1.0 $\mathrm{mL}$ of water for further use.

Formation of the MPM/MBA monolayer on the AuNPs/Au@PdNPs/Au@PtNPs A drop of the as-synthesized AuNPs/Au@PdNPs/Au@PtNPs stock solution was first deposited on precleaned Si wafer, incubated for 0.5 h. Subsequently, the nanoplates deposited $\mathrm{Si}$ wafer was immersed in a $2 \mathrm{mM}$ ethanolic MPM/MBA solution for $1 \mathrm{~h}$ to form monolayer of MPM/MBA on AuNPs/Au@PdNPs/Au@PtNPs. Finally, the modified sample was sonicated in ethanol for 3 min for removing the uncoordinated MPM/MBA molecules.

TERS Probe Fabrication Silicon AFM probes with related parameters force constant $2.7 \mathrm{~N} / \mathrm{m}$ and resonance frequency 50-80 kHz were purchased from Appnano (Mountain View, CA). Then, the metal evaporation was carried out for coating the AFM tips with a layer of gold. Briefly, two of the probes were fixed onto each of the clamped device and ten of the devices were put in the thermal evaporator chamber (MBrown, Stratham, NH). During metal deposition, the pressure was kept at $\sim 1 \times 10^{-6}$ mbar. Then, gold pellets (Kurt J. Lesker, Efferson Hills, PA) thermally evaporate at constant $0.2 \mathrm{~A} \cdot \mathrm{s}^{-1} \mathrm{rate}$. After $70 \mathrm{~nm}$ of Au was deposited on the AFM tips, the evaporation was stopped and kept cooling down to room temperature. The temperature at the tip surface and deposition chamber was $\sim 50{ }^{\circ} \mathrm{C}$.

TERS measurement AFM-TERS and AFM scanning was carried out on the AIST-NT-HORIBA system equipped with a $632.8 \mathrm{~nm}$ continuous wavelength $(\mathrm{CW})$ laser. Laser light was brought to the sample surface in a side-illumination geometry with a 100X Mitutoyo microscope objective. The scatterring electromagnetic radiation was also collected with the same objective and directed introduced to a fiber-coupled Horiba iHR550 spectrograph that equipped with a Synapse EM-CCD camera (Horiba, Edison, NJ).

SEM characterization. All the SEM images for AuNPs/Au@PdNPs/Au@PtNPs characterization were collected on a JEOL scanning electron microscope (JSM- 7500F). 
a)
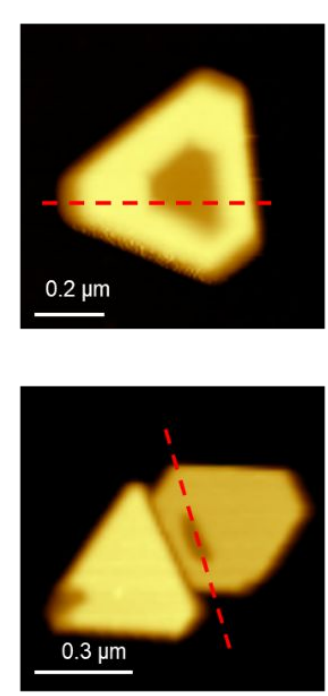

g)

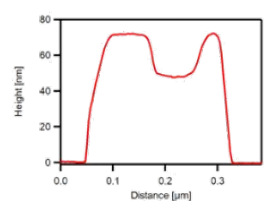

j)

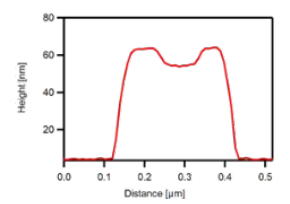

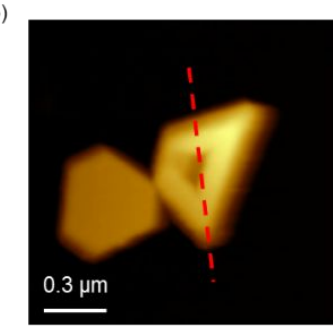

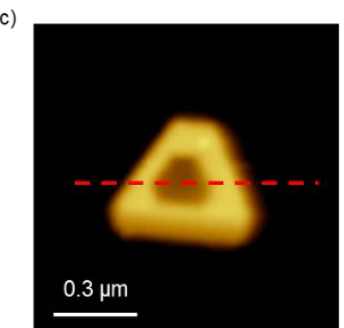

)
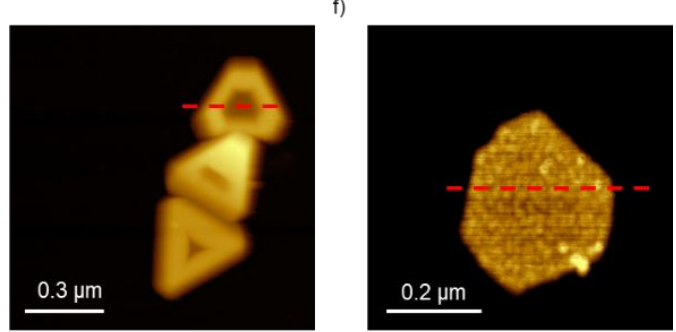

h)

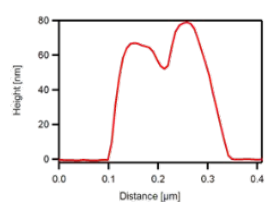

k)

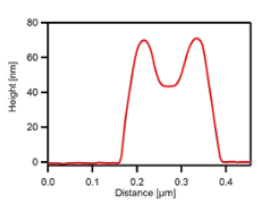

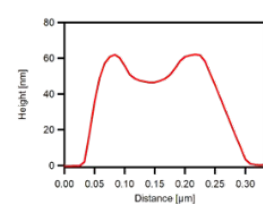

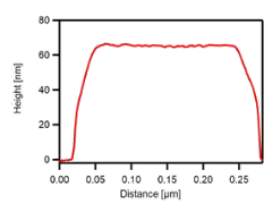

Figure S1 AFM images and height profile of Au@PtNPs. a-f AFM images of Au@PtNPs from AuNPs. g-l Height profiles of AuNPs in a-f. 
a)

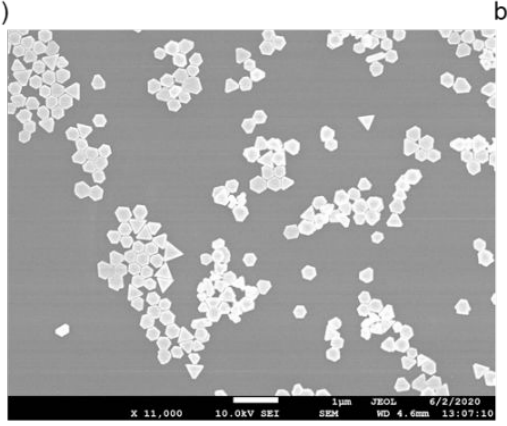

b)

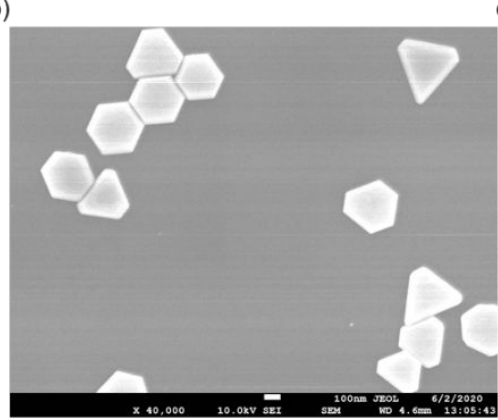

d)
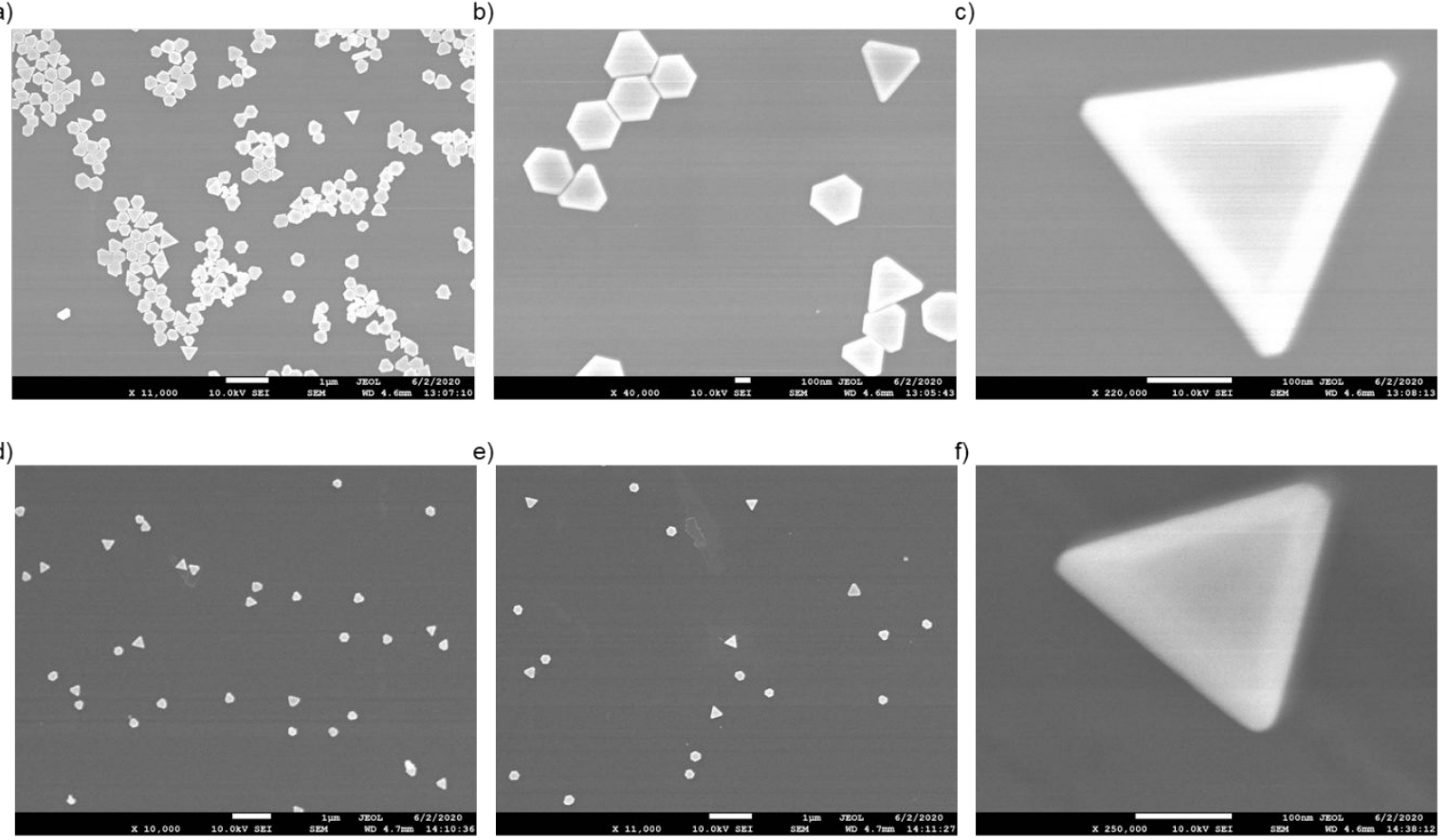

g)
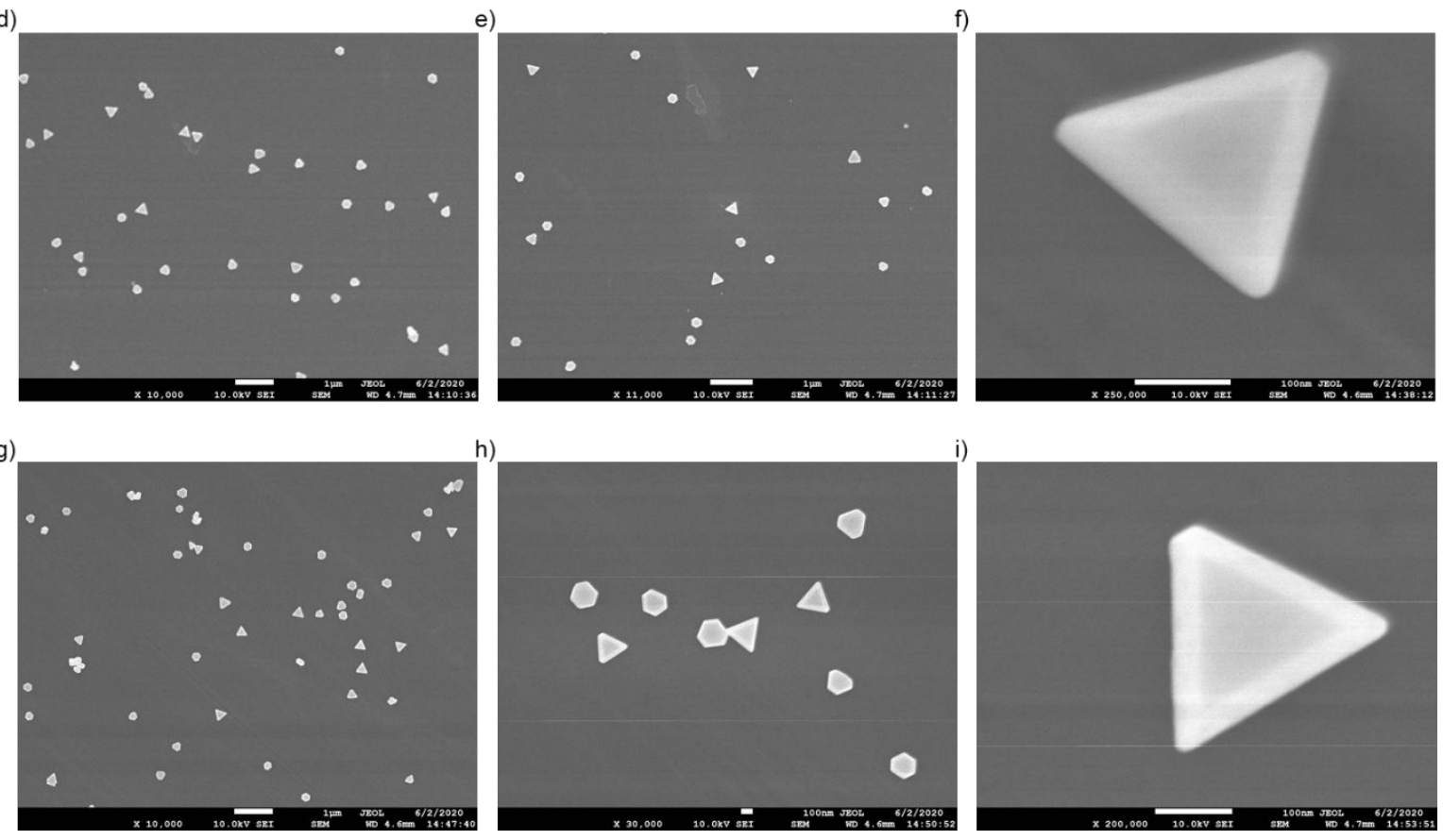

Figure S2 SEM images of AuNPs, Au@PtNPs and Au@PdNPs. a-c SEM images of AuNPs with different resolutions. d-f SEM images of Au@PtNPs with different resolutions. g-i SEM images of Au@PtNPs with different resolutions. 
a)

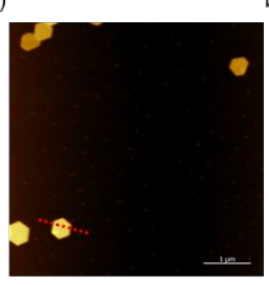

b)

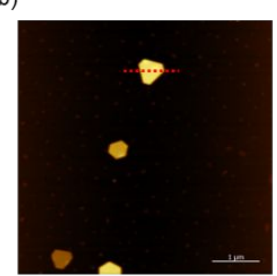

e)

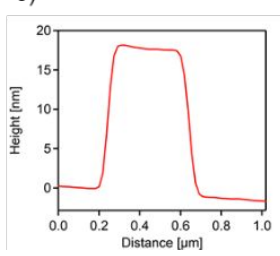

f)

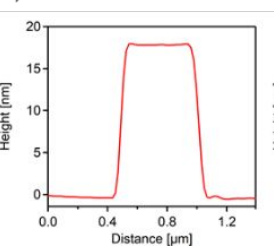

c)

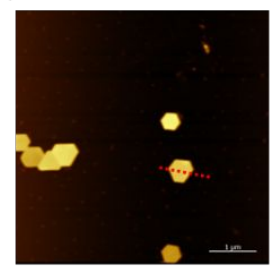

g)

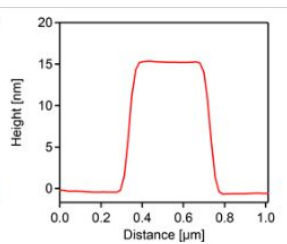

d)

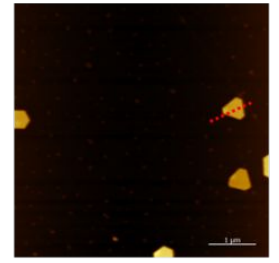

h)

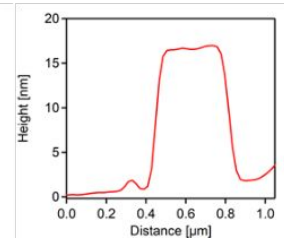

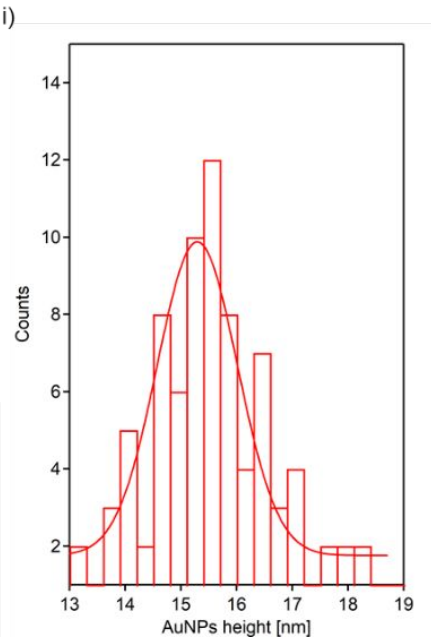

Figure S3 AFM images and height profile of 15-nm AuNPs. a-d AFM images of seed-mediated grew AuNPs. e-h Height profiles of AuNPs in a-d. i statistical analysis and gaussian fit of the AuNPs' height, the most probable height under gaussian fit was $15.4 \pm 0.8 \mathrm{~nm}$. 

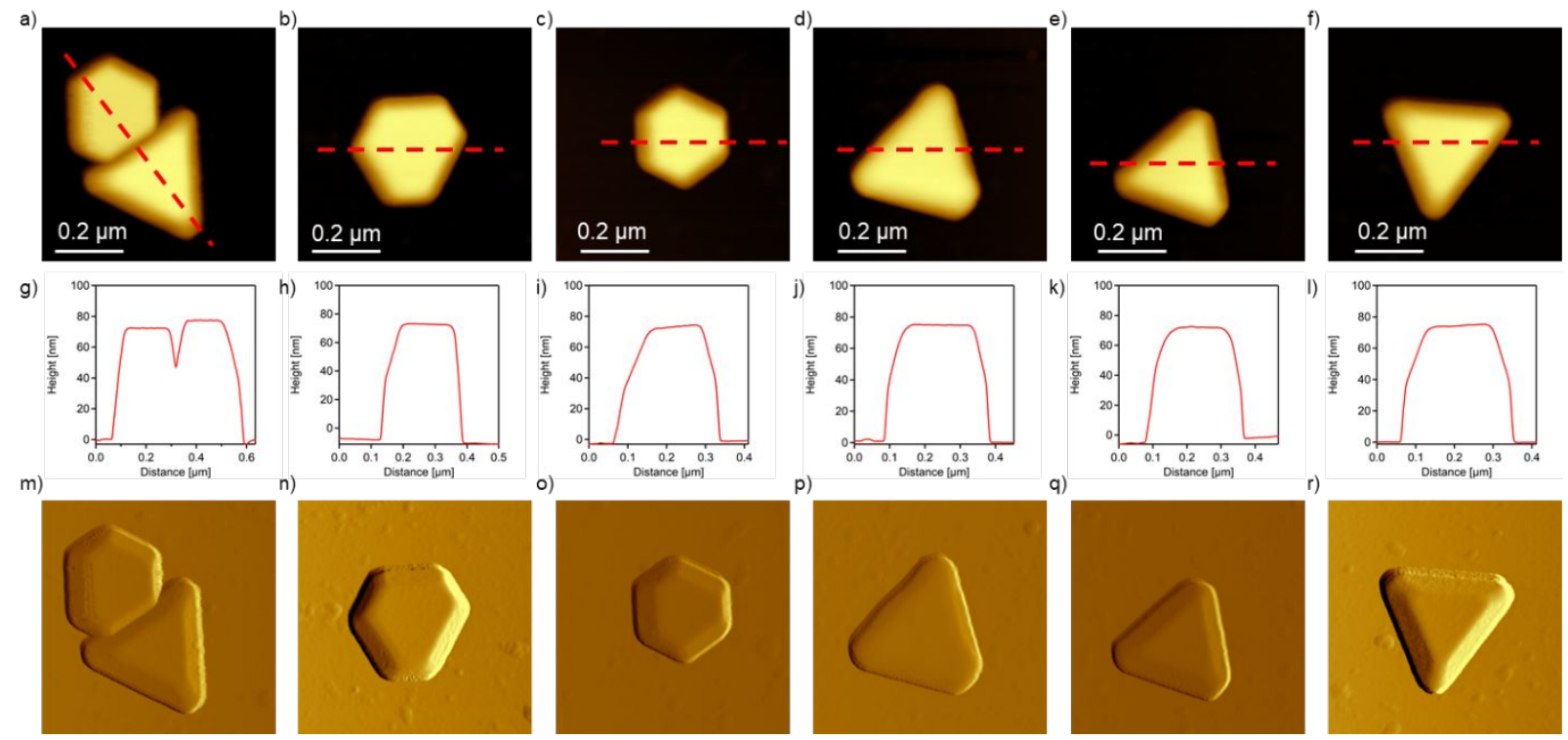

Figure S4 AFM images and height profile of thick AuNPs. a-f AFM height images of isotropical growth AuNPs from 15-nm AuNPs seeds. g-I Height porfiles of AuNPs corresponding to a-f. m-r AFM phase images of AuNPs corresponding to a-f. 

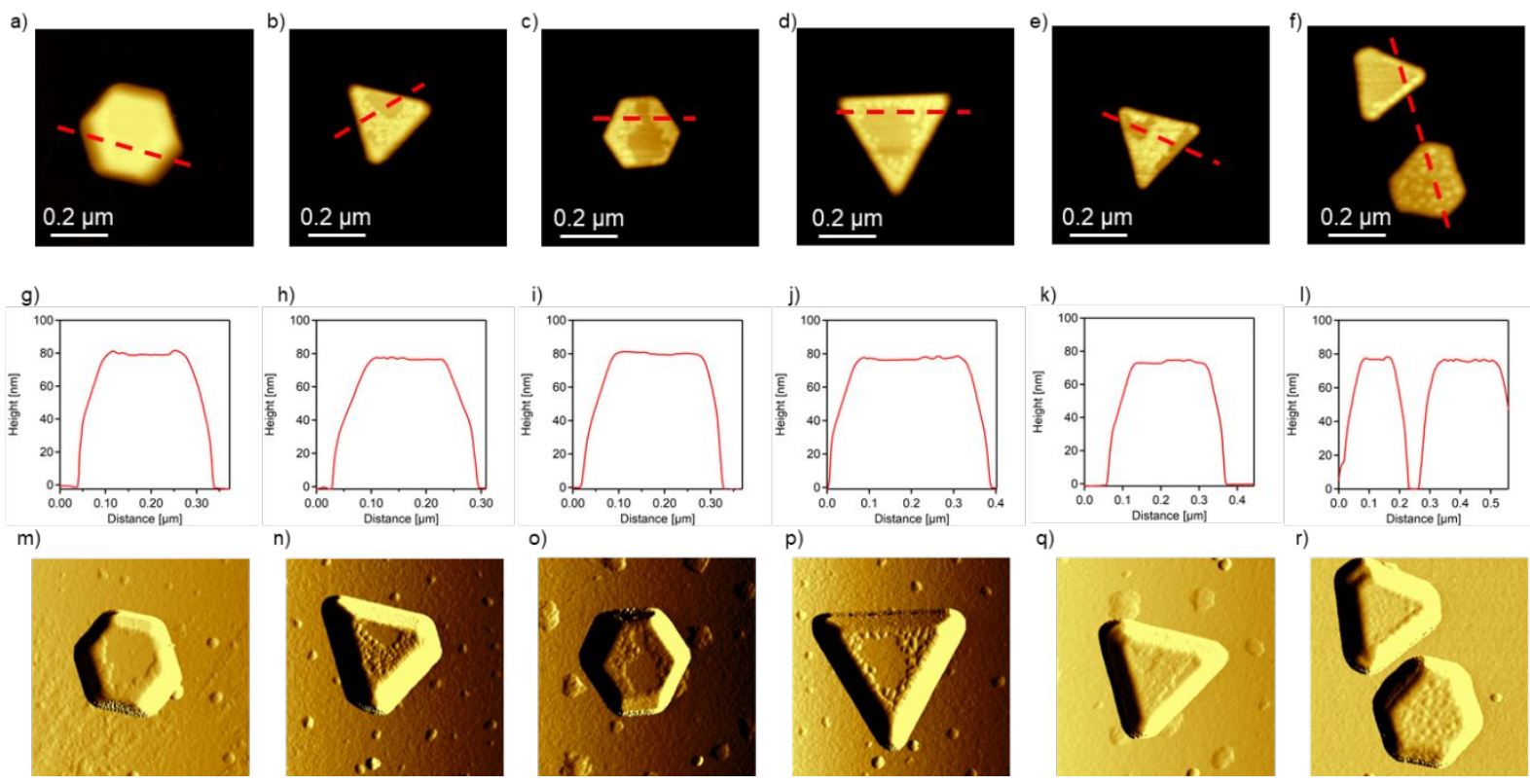

q) r)
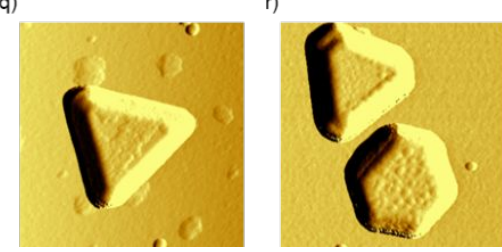

Figure S5 AFM images and height profile of Au@PdNPs. a-f AFM images of Au@PdNPs from AuNPs. g-I Height profiles of Au@PdNPs in a-f. m-r Phase mode images of Au@PdNPs corresponding to a-f. 
a)

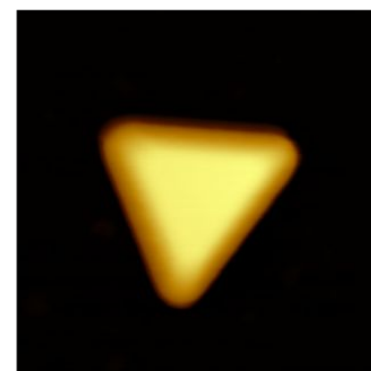

d)

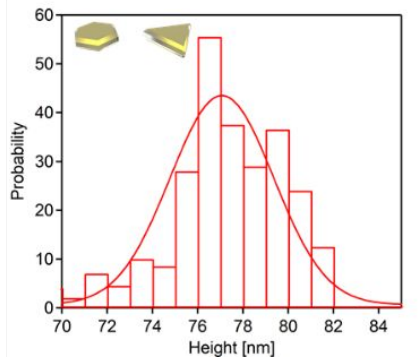

b)

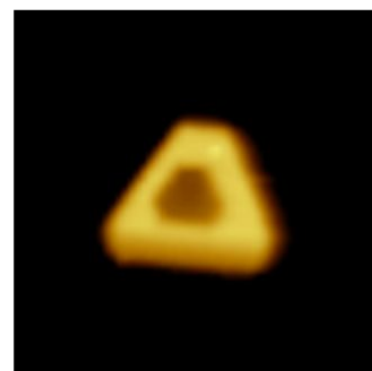

e)

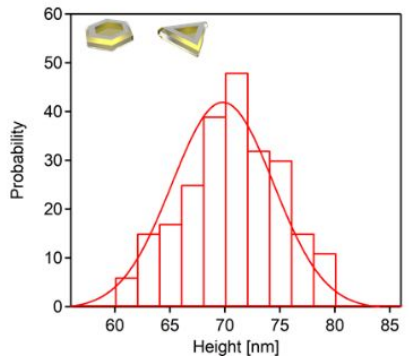

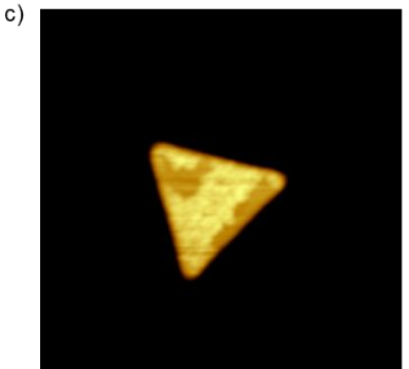

f)

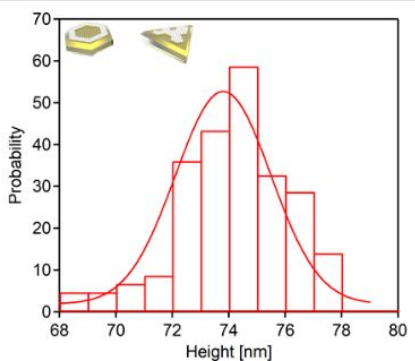

Figure S6 AFM images and statistical height analysis of Au, Au@PtNPs and Au@PdNPs. AFM images of a AuNPs b Au@PtNPs and c Au@PdNPs. Histograms and gaussian fit on d Au, e Au@PtNPs and f Au@PdNPs. The most probable height is 76.9 \pm 3.5 nm for AuNPs, $73.1 \pm 2.7$ nm for Au@PtNPs and 69.8 5.4 nm for Au@PdNPs. 


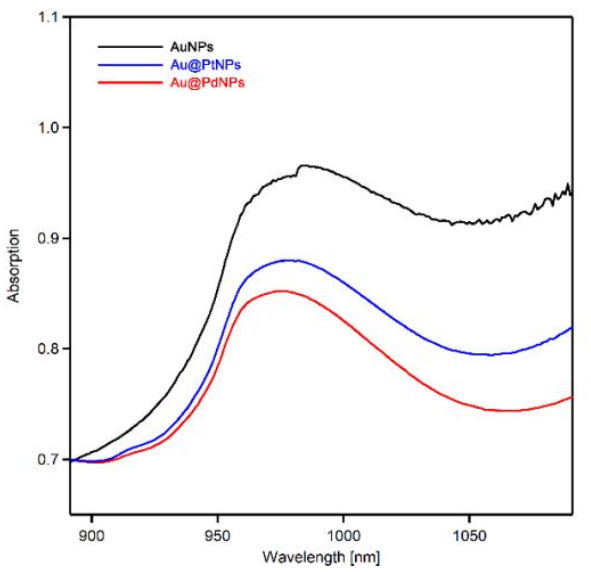

Figure S7 UV-vis-NIR of absorption spectra of AuNPs (black), Au@PtNPs (blue) and Au@PdNPs (red). 
a)

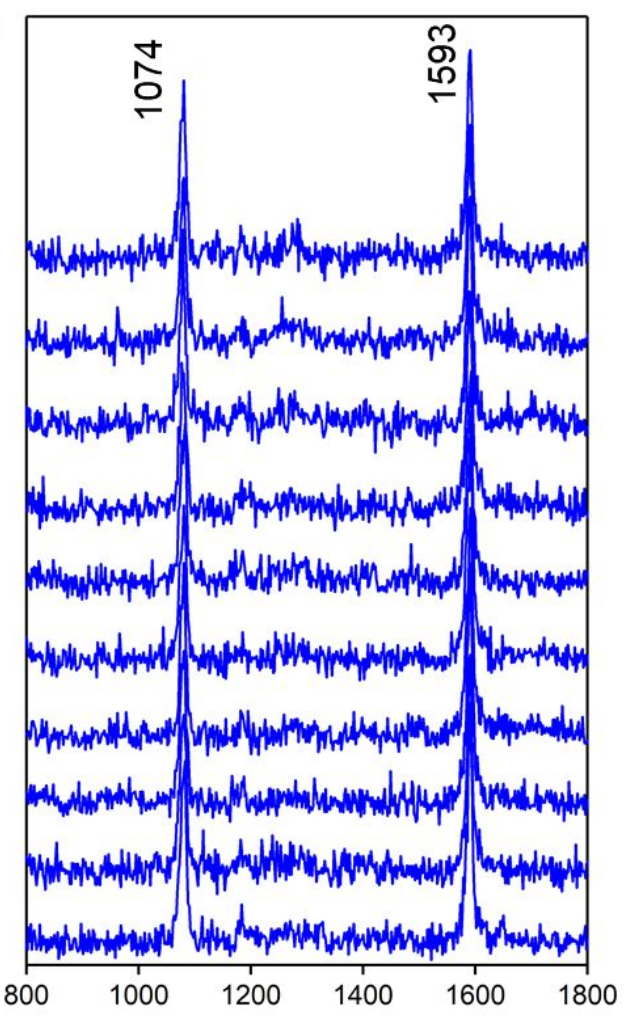

b)

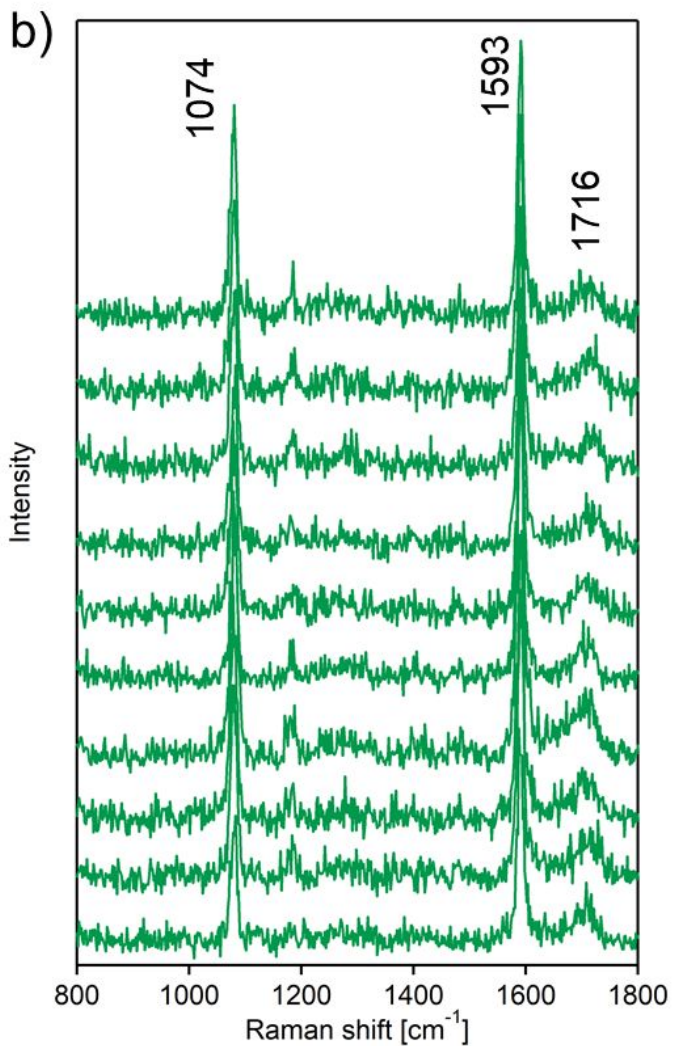

Figure S8 More TERS spectra of catalyzing MPM on Au@PtNPs. (a) TERS of MPM. (b) TERS of MBA. 
a)

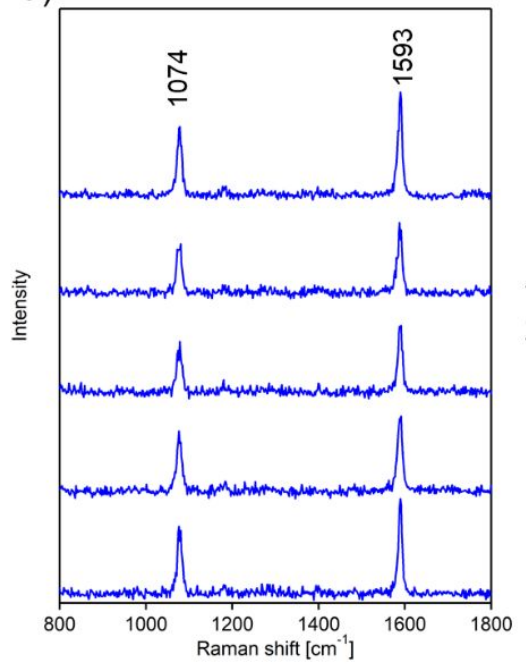

b)

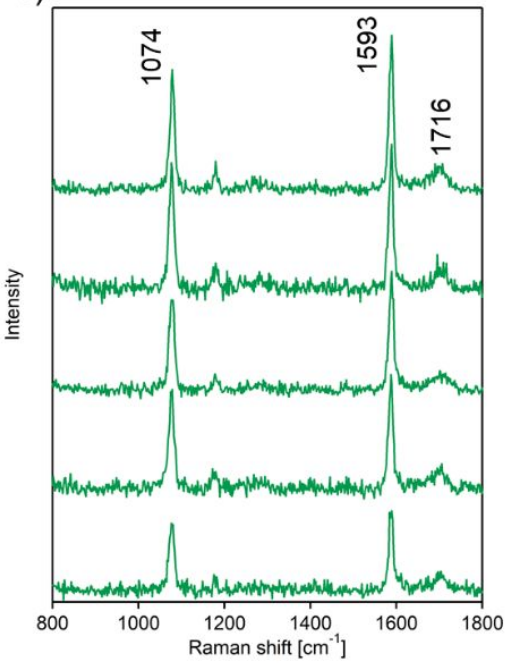

c)

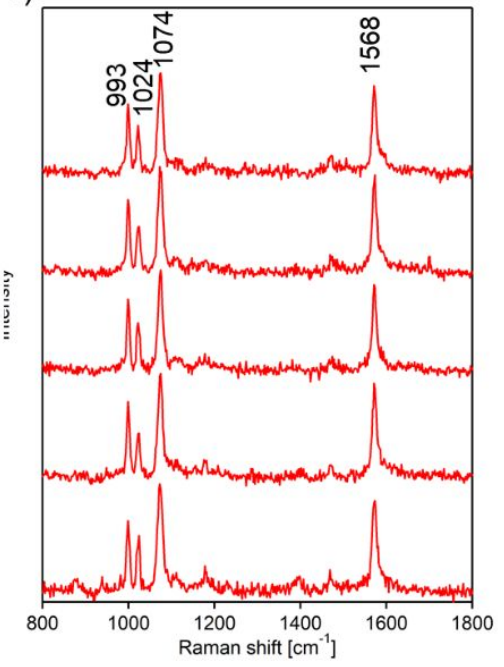

Figure S9 SERS spectra of (a) MPM, (b) MBA and (c) TP. 

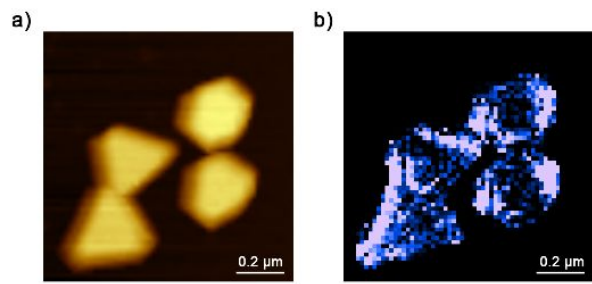

e)

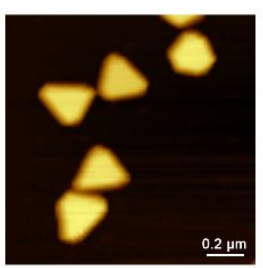

i)

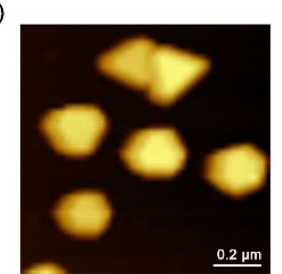

m)

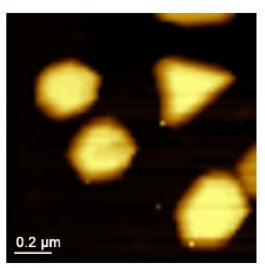

f)

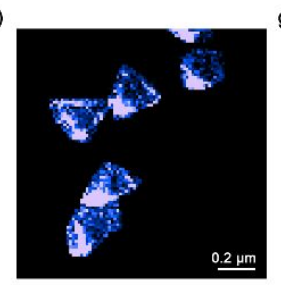

j)

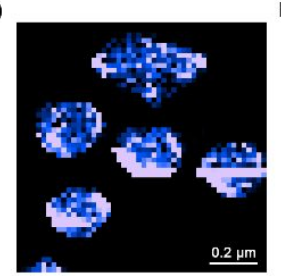

n)

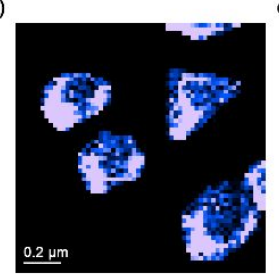

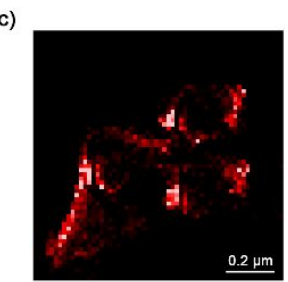

g)

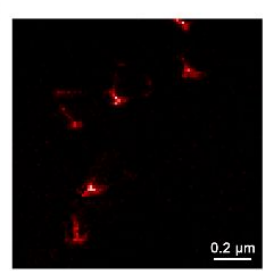

k)
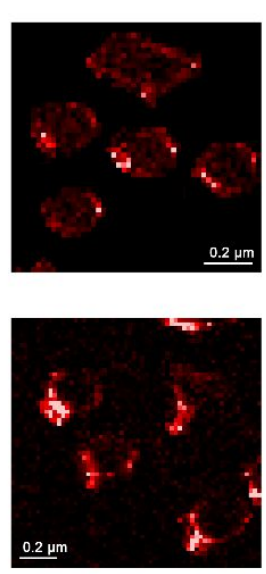

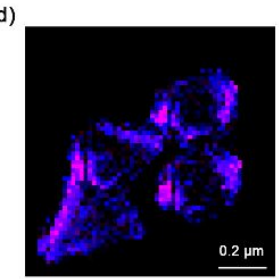

h)
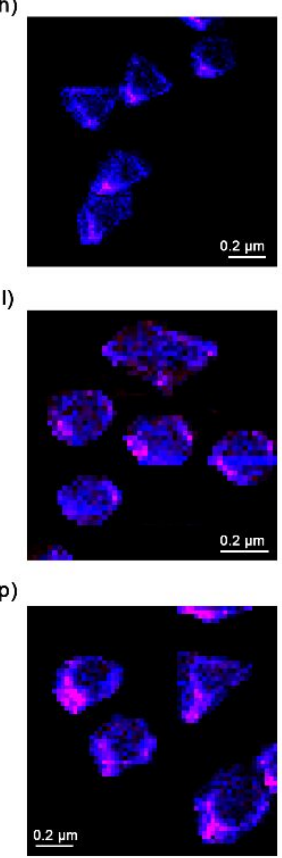

Figure S10 20-nm resolution TERS mapping of MPM/MBA on AuNPs, Au@PtNPs and Au@PdNPs. a In-situ AFM image of MPM on Au@PtNPs during TERS imaging. b,c TERS image of Au@PtNPs (20 nm per pixel). Intensity of $1593 \mathrm{~cm}^{-1}$ band (C ring vibration) of MPM is shown in blue, intensities of $1714 \mathrm{~cm}^{-1}$ (C=O vibration) of MBA is shown in red. $\mathbf{d}$ Overlapping TERS image of MPM and MBA. e In-situ AFM image of MBA on Au@PdNPs during TERS imaging. f,g TERS image of Au@PdNPs (20 nm per pixel). Intensity of $1714 \mathrm{~cm}^{-1}$ band (C=O vibration) of MBA is shown in blue, $1593 \mathrm{~cm}^{-1}$ band (C ring vibration) of MPM without showing $C=O$ vibration band is shown in red. $\mathbf{h}$ Overlapping TERS image of MPM and MBA. i In-situ AFM image of MPM on AuNPs during TERS imaging. j,k TERS image of AuNPs (20 nm per pixel). Intensity of $1593 \mathrm{~cm}^{-1}$ band (C ring vibration) of MPM is shown in blue, intensities of $1561 \mathrm{~cm}^{-1}$ (C ring vibration) of TP is shown in red. I Overlapping TERS image of MPM and TP. $\mathbf{m}$ In-situ AFM image of MBA on AuNPs during TERS imaging. $\mathbf{n}, \mathbf{o}$ TERS image of AuNPs (20 nm per pixel). Intensity of $1714 \mathrm{~cm}^{-1}$ band ( $C=O$ vibration) of MBA is shown in blue, intensities of $1561 \mathrm{~cm}^{-1}$ ( $C$ ring vibration) of TP is shown in red. $p$ Overlapping TERS image of MPM and TP. 

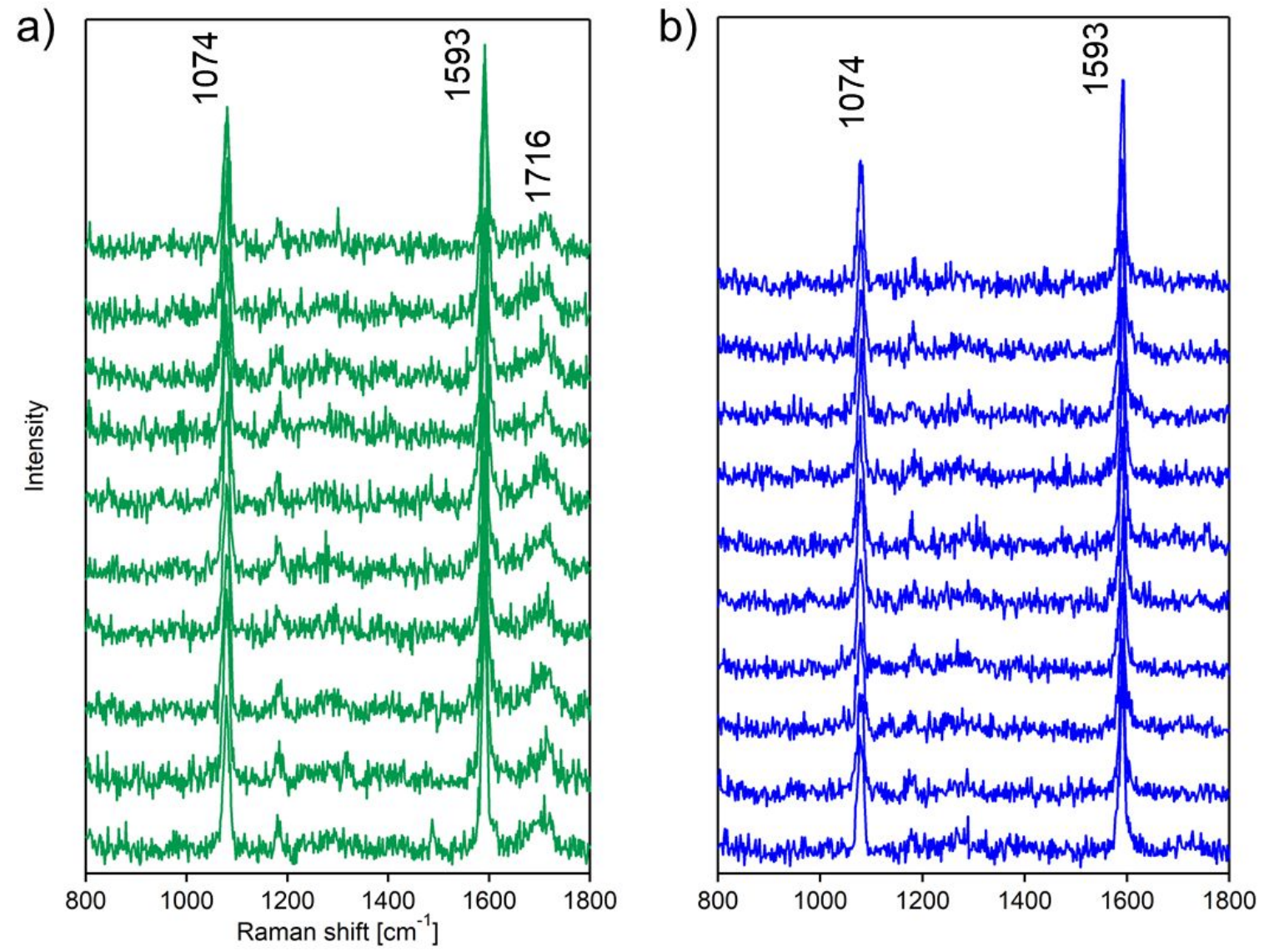

Figure S11 More TERS spectra of catalyzing MBA on Au@PdNPs. (a) TERS of MBA. (b) TERS of MPM. 
a)

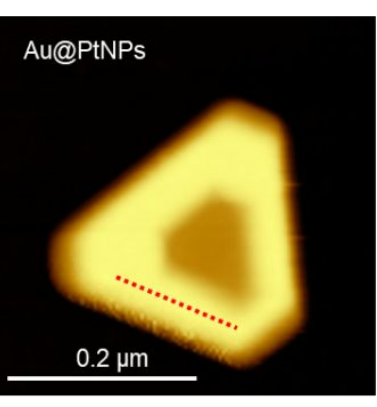

c)

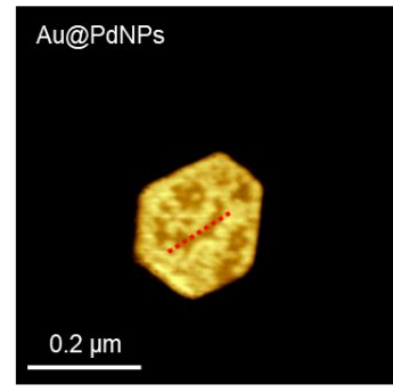

b)

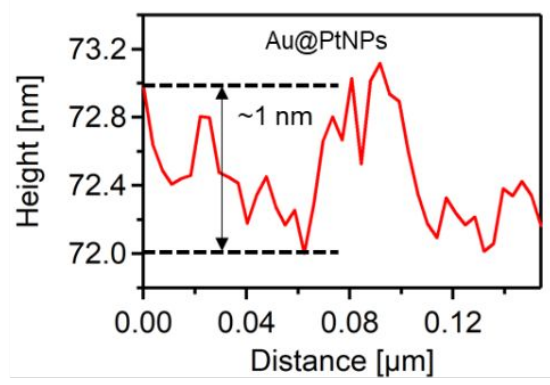

d)

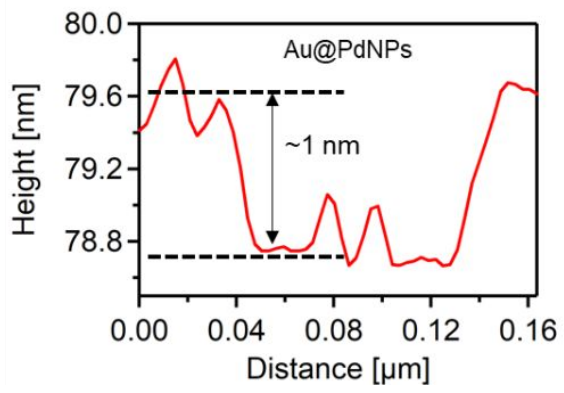

Figure S12. High resolution AFM images and height distribution of (a-b) Au@PtNPs and (c-d) Au@PdNPs. 
a)

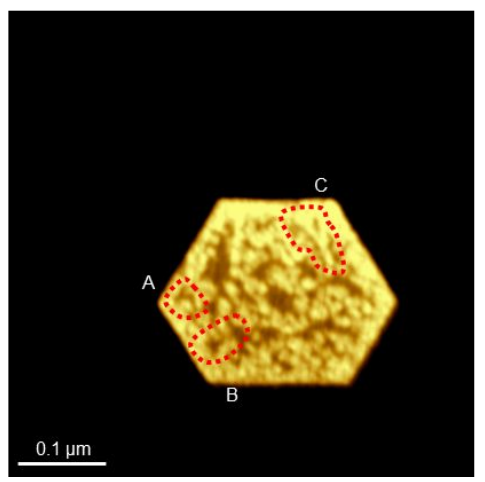

b)

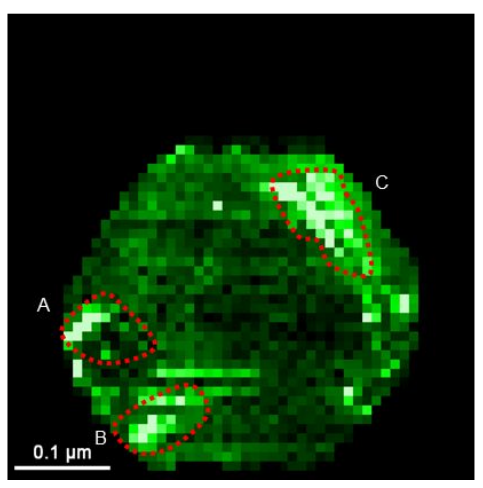

c)

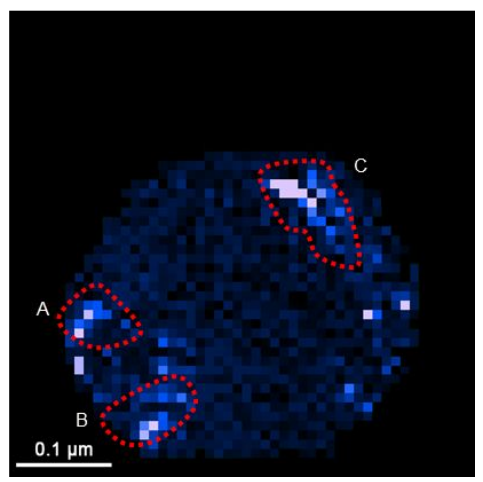

Figure S13. High resolution (a) AFM image and (b,c) TERS images of Au@PdNPs. (10 nm per pixel). Intensity of intensities of $1570-1750 \mathrm{~cm}^{-1}$ containing $C$ ring vibration and $C=O$ vibration of MBA is shown in green, $1593 \mathrm{~cm}^{-1}$ band (C ring vibration) of MPM without showing $\mathrm{C}=\mathrm{O}$ vibration band $\left(1714 \mathrm{~cm}^{-1}\right)$ is shown in blue. 

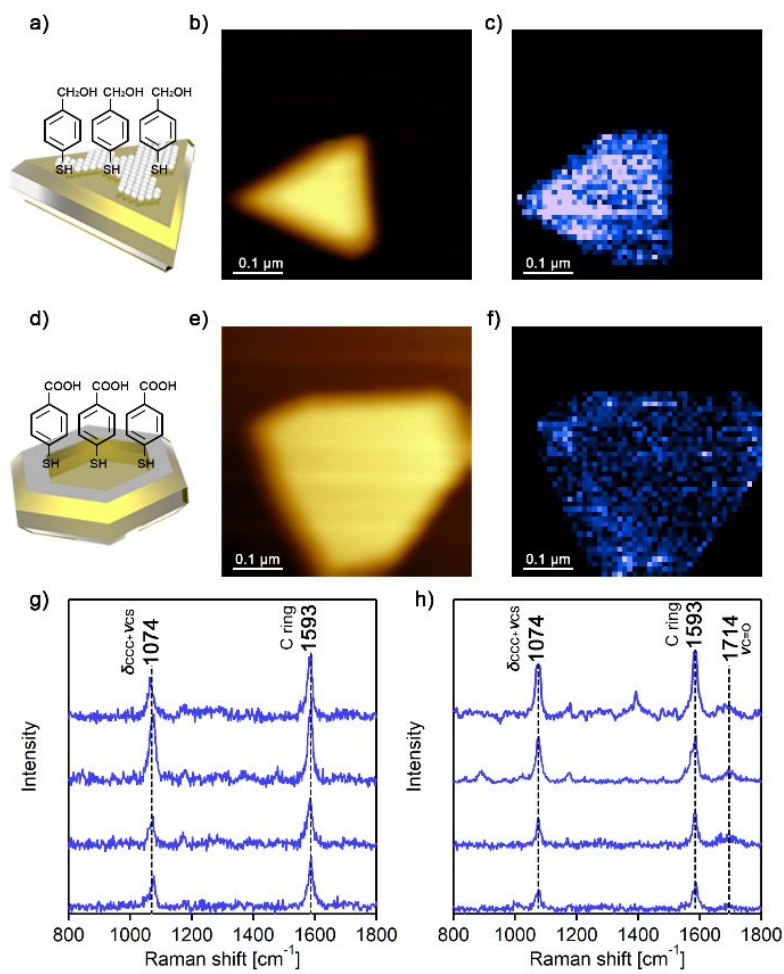

Figure S14 Control experiments showing TERS of MPM on Au@PdNPs and MBA on Au@PtNPs. a Schematic illustration of TERS image of MPM on Au@PdNPs. b In-situ AFM image of Au@PdNPs during TERS imaging. c TERS image of Au@PdNPs (10 nm per pixel). Intensity of $1593 \mathrm{~cm}^{-1}$ band (C ring vibration) of MPM is shown in blue. d Schematic illustration of TERS image of MBA on Au@PtNPs. b In-situ AFM image of Au@PtNPs during TERS imaging. c TERS image of Au@PtNPs (10 nm per pixel). Intensity of $1714 \mathrm{~cm}^{-1}$ band $(C=0)$ of MBA is shown in red. $\mathbf{g}$, $\mathbf{h}$ Typical TERS spectra extracted from chemical maps on b Au@PdNPs and e Au@PtNPs showing presence of MPM and MBA (green). 

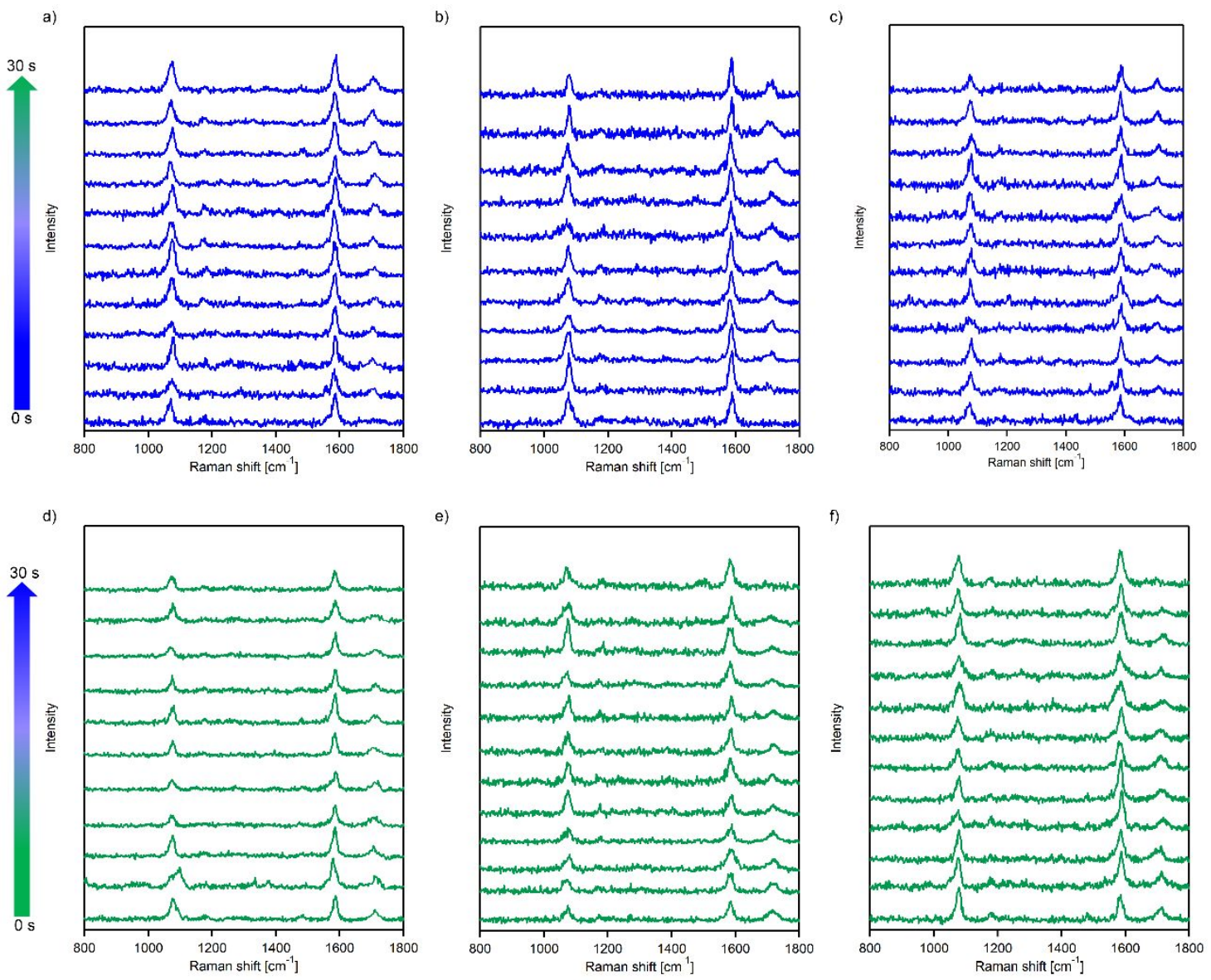

Figure S15 Kinetic catalyzation process of MPM on Au@PtNPs and MBA on Au@PdNPs. a-c MBA to MPM reduction on Au@PdNPs and d-e MPM to MBA oxidation on Au@PtNPs. 
a)

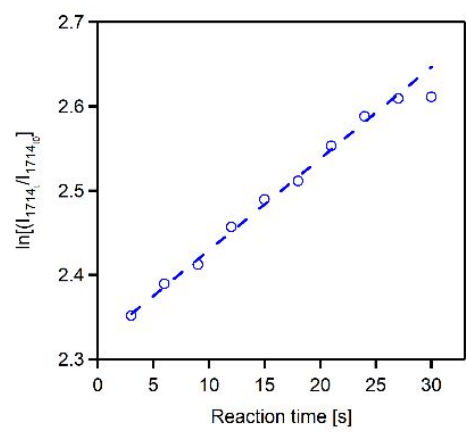

d)

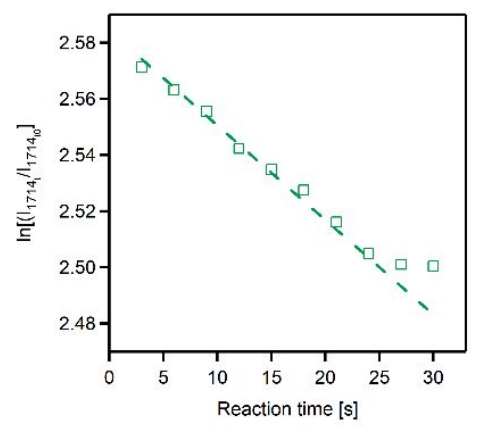

b)

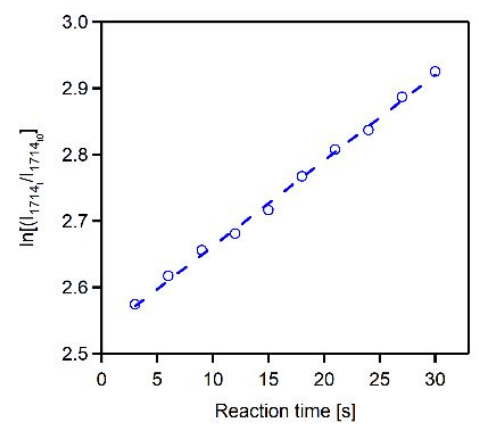

e)

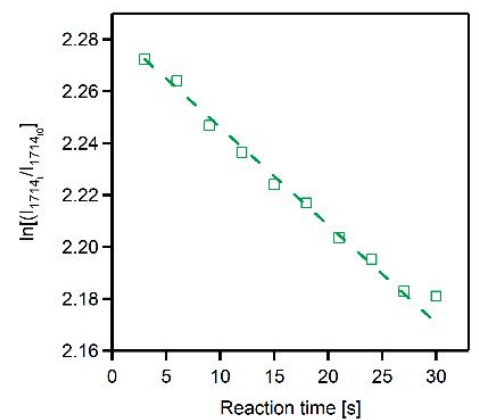

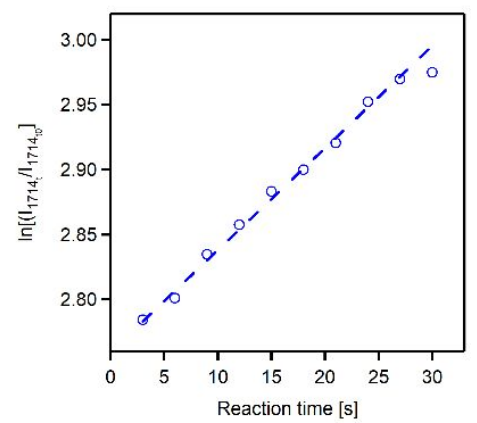

f)

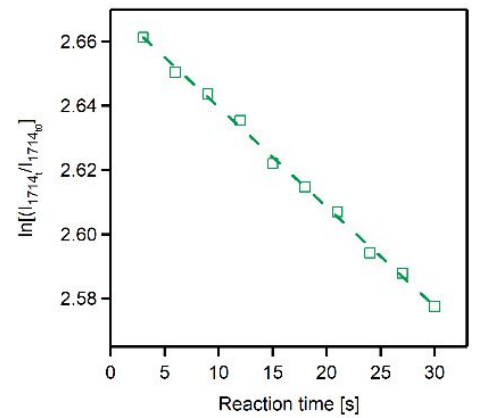

Figure S16 Rate constant of (a-c) MPM to MBA oxidation on Au@PtNPs and (d-e) MBA to MPM reduction on Au@PdNPs at different spots. 

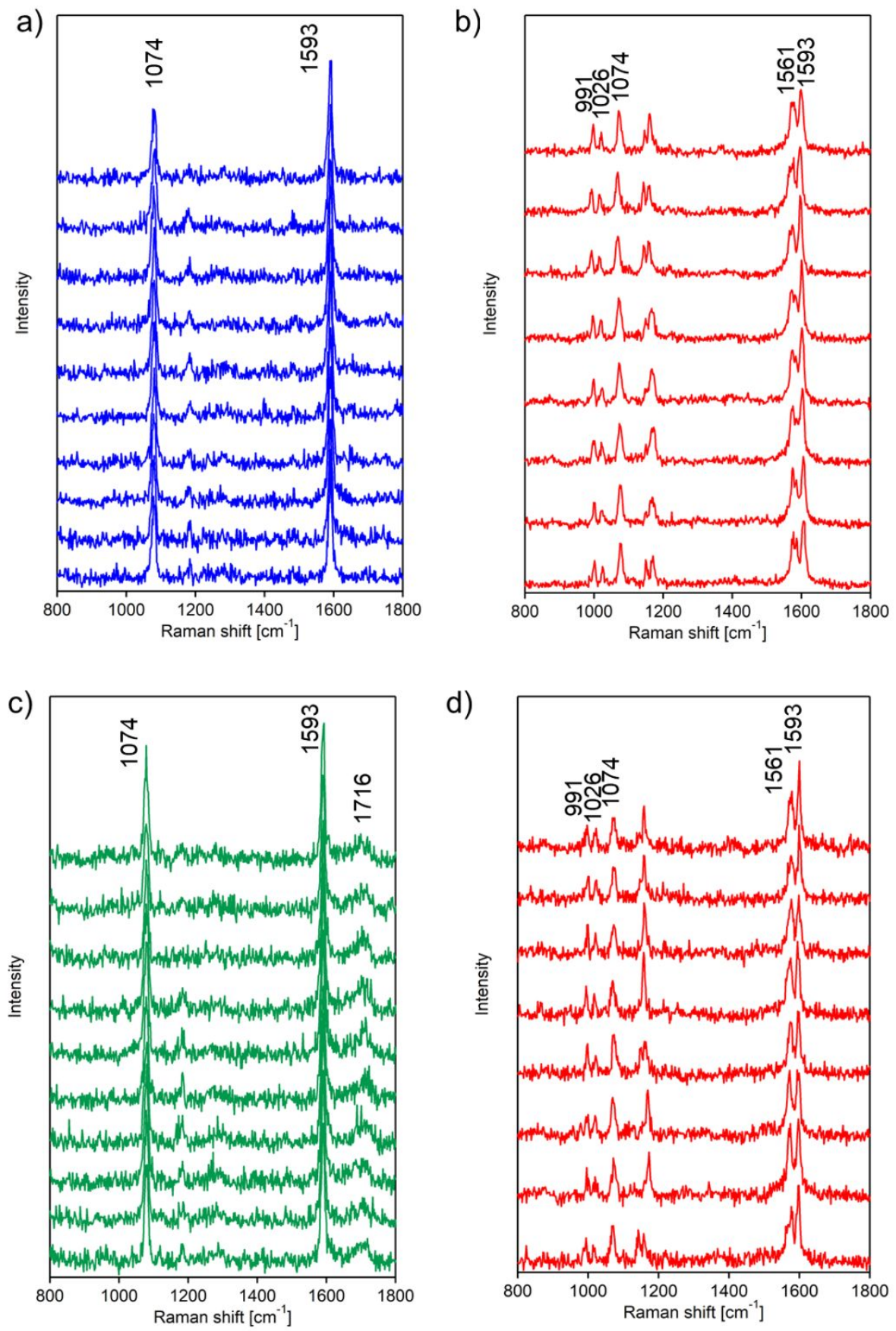

Figure S17 More TERS spectra of catalyzing (a) MPM on AuNPs to (b) TP and (c) MBA to (d) TP. 
Table S1. Kinetic parameters of MPM oxidation and MBA reduction.

Table S1 Kinetic parameters of MPM oxidation and MBA reduction

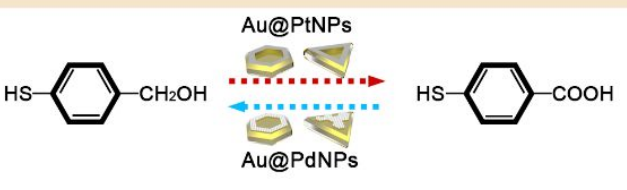

rate constant of rate constant of MPM oxidation MBA reduction

$\begin{array}{lll}1 & 9.5 \times 10^{-3} \mathrm{~s}^{-1} & 2.7 \times 10^{-3} \mathrm{~s}^{-1} \\ 2 & 1.1 \times 10^{-2} \mathrm{~s}^{-1} & 3.4 \times 10^{-3} \mathrm{~s}^{-1} \\ 3 & 1.3 \times 10^{-2} \mathrm{~s}^{-1} & 3.8 \times 10^{-3} \mathrm{~s}^{-1} \\ 4 & 7.9 \times 10^{-3} \mathrm{~s}^{-1} & 3.1 \times 10^{-3} \mathrm{~s}^{-1}\end{array}$

